\title{
Multiple Pulmonary Arteriovenous Fistula in a Mother and His Son: A Case Report
}

Ata Firouzi*, Soudabe Behrooj, Mohsen Neshati Pirborj, Majid Abedinejad and Omid Shafe

Cardiovascular Intervention Research Center, Rajaie Cardiovascular Medical and Research Center, Iran University of Medical Sciences, Tehran, Iran

\begin{abstract}
Multiple Pulmonary Arteriovenous Fistulas (PAVFs) are rare vascular abnormalities in the general population, with a report on 15000 autopsies revealing only 3 cases of multiple PAVFs. However, these abnormal communications between pulmonary arteries and veins are commonly seen in patients with Hereditary Hemorrhagic Telangiectasia (HHT). Several conditions such as cirrhosis, chest trauma, metastatic carcinoma, schistosomiasis, and systemic amyloidosis are accountable for acquired form PAVF. We describe 2 patients (mother and son) with multiple PAVFs and no apparent signs or symptoms suggestive of HHT. A pulmonary artery injection wes performed for Case 1 (mother) and showed bilateral PAVFs with aneurysmal dilation. Given the diagnosis of PAVFs in the mother, we considered the same pathology for the child's symptoms. Therefore, the boy also underwent pulmonary angiography, which revealed multiple bilateral PAVFs. Both patients underwent interventional angiography and the PAVFs were occulted with stainless coil. In conclusion, the presence of multiple PAVFs should be considered in the differential diagnosis of patients with unexplained exertional dyspnea and cyanosis, even in the absence of diagnostic features suggestive of HHT.
\end{abstract}

Keywords: Bilateral PAVFs; Exertional dyspnea

\section{Introduction}

Multiple pulmonary arteriovenous fistulas (PAVFs) are rare vascular abnormalities in the general population, with a report on 15000 autopsies revealing only 3 cases of multiple PAVFs [1]. However, these abnormal communications between pulmonary arteries and veins are commonly seen in patients with hereditary hemorrhagic telangiectasia (HHT) (also known as the Osler-Weber-Rendu syndrome) [2,3]. Aside from the congenital etiology, several conditions may lead to acquired forms of multiple PAVFs-including cirrhosis, chest trauma, metastatic carcinoma, schistosomiasis, and systemic amyloidosis $[4,5]$. The most common clinical symptom of multiple PAVFs is dyspnea on exertion, reported in $13 \%$ to $55 \%$ of patients in different reports [5-7]. We describe 2 patients (mother and son) with multiple PAVFs and no apparent signs or symptoms suggestive of HHT.

\section{Case Reports}

A 26-year-old female (Case 1) and her 8-year-old son (Case 2) were referred to our clinic with complaints of dyspnea on exertion and mild chest pain. The mother reported several episodes of cyanosis of lips and fingers following physical activity. She had begun experiencing these symptoms 4 years previously. Neither the mother nor the child had a history of chest trauma or surgery, melena, or epistaxis. Both patients were admitted to our center and underwent full physical and clinical examinations.

\section{Case 1}

The physical examination of the mother was unremarkable, and no telangiectasia on her skin was noticeable. Heart and lungs auscultations were normal. ECG showed a normal sinus rhythm, and chest radiograph demonstrated a prominent pulmonary artery, a round lesion in the right lung, and a tubular lesion in the left lung (Figure 1). Fundoscopic examination was normal, and no sign of telangiectasia was noted in her conjunctiva. Transthoracic echocardiography showed a normal left ventricle size with a preserved systolic function (ejection fraction $=50 \%$ to $55 \%$ ) and a normal right atrium size and function with no abnormal findings. An angiogram was scheduled in order to further assess the presence of structural abnormalities. A pulmonary artery

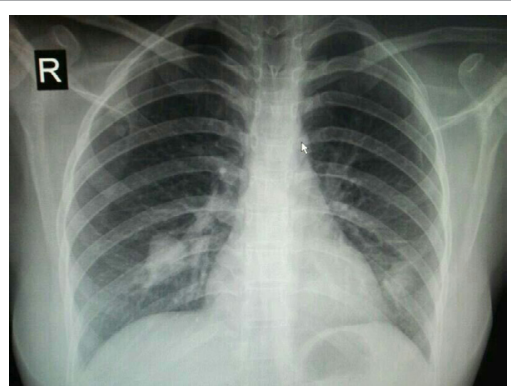

Figure 1: Chest radiograph of case 1, showing a prominent pulmonary artery, a low-density round lesion in the lower lobe of the right lung, and a tubular lesion in the lower lobe of the left lung.

injection showed bilateral PAVFs with aneurysmal dilation (Figure 2). Laboratory tests were ordered in order to rule out the known causes of multiple PAVFs such as HHT, cirrhosis, and amyloidosis. She had normal liver function tests with negative viral marker tests (hepatitis B and C), normal serum protein electrophoresis, normal complete blood count, and normal thyroid function tests.

The patient underwent interventional angiography for the treatment of the PAVFs in the left lung. After catheterizing the feeding artery of 2 PAVFs in the left pulmonary area, we passed through one $7 \mathrm{~mm} \times 6$ $\mathrm{mm}$ stainless steel coil and two $9 \mathrm{~mm} \times 6 \mathrm{~mm}$ coils and deployed them to occlude the PAVFs. She was discharged with a prescription of aspirin

*Corresponding author: Ata Firouzi, Cardiovascular Intervention Research Center, Rajaie Cardiovascular Medical and Research Center, Iran University of Medical Sciences, Tehran, Iran, Tel: 982123922174; Fax: 982122663217; E-mail: atafirouzi@yahoo.com

Received May 24, 2017; Accepted June 29, 2017; Published July 04, 2017

Citation: Firouzi A, Behrooj S, Pirborj MN, Abedinejad M, Shafe O (2017) Multiple Pulmonary Arteriovenous Fistula in a Mother and His Son: A Case Report. J Cardiovasc Dis Diagn 5: 285. doi: 10.4172/2329-9517.1000285

Copyright: ( 2017 Firouzi A, et al. This is an open-access article distributed under the terms of the Creative Commons Attribution License, which permits unrestricted use, distribution, and reproduction in any medium, provided the original author and source are credited. 

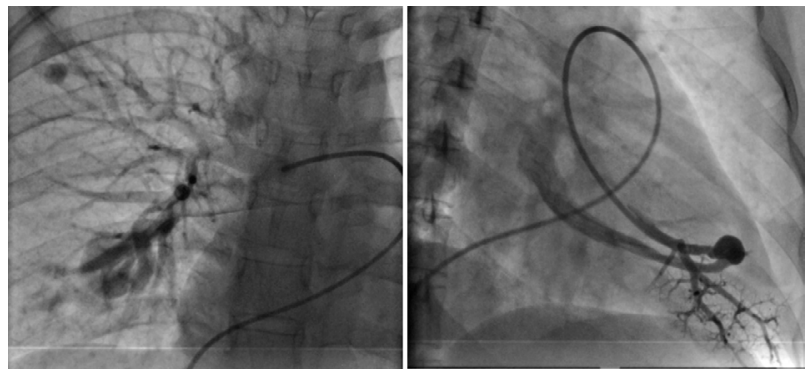

Figure 2: Angiogram of case 1, showing multiple pulmonary arteriovenous fistulas in both lungs.
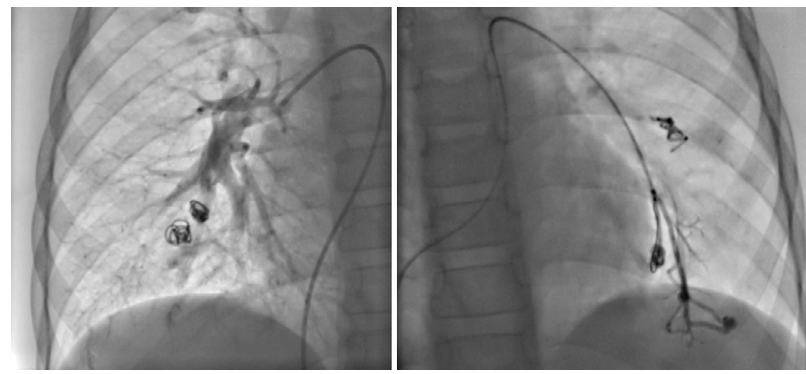

Figure 3: Angiogram of case 2 after embolization, demonstrating multiple pulmonary arteriovenous fistulas in the lower lobes of the left and right lungs occluded with a coil.

$(80 \mathrm{mg} / \mathrm{d})$, and a second angiographic procedure was scheduled for the following month for the embolization of the PAVFs in the right lung. During the second angiography, a left pulmonary injection showed the complete occlusion of the left PAVFs with the coil. We occluded the PAVF in the middle lobe of the right lung with 1 steel coil $(9 \mathrm{~mm} \times 6$ $\mathrm{mm}$ ). The follow-up visits in the upcoming weeks showed the resolution of the patient's symptoms.

\section{Case 2}

Similar to Case 1-all physical, clinical, and laboratory examinations were unremarkable for the known causes of multiple PAVFs. Transthoracic echocardiography showed no atrial or ventricular septal defects or patent ductus arteriosus. Given the diagnosis of PAVFs in the mother, we considered the same pathology for the child's symptoms. Therefore, the boy also underwent pulmonary angiography, which revealed multiple bilateral PAVFs (Figure 3). We occluded 2 fistulas in the lower lobe of the left lung using 2 steel coils $(7 \mathrm{~mm} \times 6 \mathrm{~mm})$ and raised the $\mathrm{O}_{2}$ saturation of the peripheral blood samples to $92 \%$ after the intervention. The patient was discharged, and a second interventional angiographic procedure was planned for the following month. During the second procedure, 4 large PAVFs in the right lung and 2 residual fistulas in the left lung were detected. We successfully occluded 2 PAVFs in the lower lobe of the right lung using 3 steel coils $(7 \mathrm{~mm} \times 6 \mathrm{~mm})$. Re-intervention for the closure of the multiple residual fistulas in the left lung and the middle lobe of the right lung were scheduled for the near future.

\section{Discussion}

According to the literature, the main congenital cause of multiple PAVFs is HHT. Believed to be the result of sporadic mutations in nearly $20 \%$ of cases with no positive family history, HHT is an autosomal dominant disease [8]. The definite diagnosis of this condition is established when a patient has 3 of the following signs and symptoms: telangiectasia, epistaxis, visceral lesion, and family history [8]. The first 2 manifestations are the most commonly reported presentations; they were, however, not observed in our patients. Although the presence of multiple PAVFs in a mother and her son suggests a hereditary cause, the absence of the known symptoms prompted us not to consider HHT as the underlying cause. Another uncommon disease linked to pulmonary artery malformations is Behçet's disease, with its highest prevalence rate reported from countries near the ancient Silk Road [9]. Patients with Behçet's disease may develop pulmonary involvement in up to $7 \%$ of the cases [10]. The most common complication is a pulmonary aneurysm, which in some cases gives rise to massive hemoptysis [11] Other complications include arterial and venous thromboses, recurrent pneumonia, and arteriobronchial fistulas [12].

Several diagnostic modalities can be used to detect PAVFsincluding the $100 \%$ oxygen method, radionuclide imaging, and contrast echocardiography [4]. However, angiography remains the gold standard for diagnosis. In the $100 \%$ oxygen method, the patient breathes $100 \%$ oxygen for at least 20 minutes and by measuring end capillary $\left(\mathrm{Cco}_{2}\right)$, arterial $\left(\mathrm{CaO}_{2}\right)$, and venous $\left(\mathrm{Cvo}_{2}\right)$ oxygen contents, the shunt fraction is calculated using the shunt equation $\left(\left(\mathrm{CCO}_{2}-\mathrm{CaO}_{2}\right) /\right.$ $\left.\left(\mathrm{CCO}_{2}-\mathrm{CVO}_{2}\right)\right)$ [13]. If the shunt fraction exceeds $5 \%$, further diagnostic tests are recommended. This method may be inexpensive, but it has lower specificity than other techniques. Contrast echocardiography requires an injection of agitated saline into a peripheral vein. In healthy individuals, the created bubbles are trapped in the lungs-whereas in patients with PAVFs, the bubbles are immediately seen in the left atrium via transthoracic echocardiography [14]. Radionuclide imaging can also be employed to measure the shunt fraction. The main disadvantage of this method is its inability to differentiate between the pulmonary and cardiac origins of the shunt [15].

Transcatheter embolization is the preferred method of treatment for multiple PAVFs and has virtually obviated the need for surgical intervention. Catheterization is performed via the femoral vein; the feeding artery of PAVFs is catheterized and steel coils or detachable balloons are advanced through the catheter [4]. It is recommended that pulmonary angiography be repeated to ensure the proper occlusion of PAVFs [16]. This technique is safe and effective-as indicted by Lee et al. [17], who reported 45 patients with 52 PAVFs subjected to embolotherapy. The authors also reported that although angina and paradoxical emboli were observed in some patients, none of them resulted in permanent sequelae. In addition, repeated embolization was needed in 8 patients for the treatment of persistent PAVFs or recanalization.

\section{Conclusion}

In conclusion, the presence of multiple PAVFs should be considered in the differential diagnosis of patients with unexplained exertional dyspnea and cyanosis, even in the absence of diagnostic features suggestive of HHT. Transcatheter embolization is a safe and effective technique for the management of this condition.

\section{References}

1. Sloan RD, Cooley RN (1953) Congenital pulmonary arteriovenous aneurysm Am J Roentgenol Radium Ther Nucl Med 70: 183-210.

2. Parra JA, Cuesta JM, Zarrabeitia R, Farinas-Alvarez C, Bueno J, et al. (2016) Screening pulmonary arteriovenous malformations in a large cohort of Spanish patients with hemorrhagic hereditary telangiectasia. Int J Cardiol 218: 240-245.

3. Cottin V, Chinet T, Lavole A, Corre R, Marchand E, et al. (2007) Pulmonary arteriovenous malformations in hereditary hemorrhagic telangiectasia: A series of 126 patients. Medicine $86: 1-17$. 
Citation: Firouzi A, Behrooj S, Pirborj MN, Abedinejad M, Shafe O (2017) Multiple Pulmonary Arteriovenous Fistula in a Mother and His Son: A Case Report. J Cardiovasc Dis Diagn 5: 285. doi: 10.4172/2329-9517.1000285

Page 3 of 3

4. Iqbal M, Rossoff LJ, Steinberg HN, Marzouk KA, Siegel DN (2000) Pulmonary arteriovenous malformations: A clinical review. Postgrad Med J 76: 390-394.

5. Khurshid I, Downie GH (2002) Pulmonary arteriovenous malformation. Postgrad Med J 78: 191-197.

6. Dines DE, Arms RA, Bernatz PE, Gomes MR (1974) Pulmonary arteriovenous fistulas. Mayo Clinic proceedings 49: 460-465.

7. Dines DE, Seward JB, Bernatz PE (1983) Pulmonary arteriovenous fistulas. Mayo Clinic proceedings 58: 176-181.

8. Khoja AM, Jalan RK, Jain DL, Kajale OV (2016) Osler-Weber-Rendu disease: A rare cause of recurrent hemoptysis. Lung India 33: 313-316.

9. Zouboulis CC (1999) Epidemiology of Adamantiades-Behcet's disease. Ann Med Interne 150: 488-498.

10. Erkan F (1999) Pulmonary involvement in Behcet disease. Curr Opin Pulm Med 5: 314-318

11. Iscan ZH, Vural KM, Bayazit M (2005) Compelling nature of arterial manifestations in Behcet disease. J Vasc Surg 41: 53-58.
12. Erkan F, Gul A, Tasali E (2001) Pulmonary manifestations of Behcet's disease. Thorax 56: 572-578

13. Chiang ST (1968) Anomogram for venous shunt (Qs-Qt) calculation. Thorax 23: $563-565$.

14. Barzilai B, Waggoner AD, Spessert C, Picus D, Goodenberger D (1991) Two-dimensional contrast echocardiography in the detection and followup of congenital pulmonary arteriovenous malformations. Am J Cardiol 68: 1507-1510.

15. Whyte MK, Peters AM, Hughes JM, Henderson BL, Bellingan GJ, et al. (1992) Quantification of right to left shunt at rest and during exercise in patients with pulmonary arteriovenous malformations. Thorax 47: 790-796.

16. White RI, Pollak JS, Wirth JA (1996) Pulmonary arteriovenous malformations: Diagnosis and transcatheter embolotherapy. J Vasc Interv Radiol 7: 787-804.

17. Lee DW, White RI, Egglin TK, Pollak JS, Fayad PB, et al. (1997) Embolotherapy of large pulmonary arteriovenous malformations: Long-term results. Ann Thorac Surg 64: 930-940. 